\title{
Characterization of the Population Pharmacokinetics of Moxidectin in Adults Infected with Strongyloides Stercoralis: Support for a Fixed-Dose Treatment Regimen
}

\author{
Cornelis Smit ${ }^{1}$ - Daniela Hofmann ${ }^{2,3} \cdot$ Somphou Sayasone $e^{2,4} \cdot$ Jennifer Keiser ${ }^{2,3} \cdot$ Marc Pfister $^{1,5}$
}

Accepted: 5 June 2021 / Published online: 23 July 2021

(c) The Author(s) 2021

\begin{abstract}
Background Moxidectin has recently attracted attention as a novel candidate for the treatment of helminth infections, including Strongyloides stercoralis. This study aims to characterize the population pharmacokinetics (PPK) of moxidectin in $S$. stercoralis-infected adults using a pharmacometric approach, and to perform model-based simulations to explore different drug dosing strategies.

Methods A PPK study embedded in a dose-escalation phase IIa trial was conducted in NamBak, Laos. Eight micro blood samples were collected from each of 96 S. stercoralis-infected adults following a moxidectin dose-ranging study, from 2 to $12 \mathrm{mg}$. A PPK model was developed using nonlinear mixed-effects modeling, and dosing strategies were explored using simulations in S. stercoralis-infected subjects with varying age and body weight ( $n=5000$ per dosing strategy).

Results A two-compartment model including delayed absorption with lag-time best described the available PK data. Allometric scaling was applied to account for the influence of body weight. High clearance was found in the infected adults (4.47 $\mathrm{L} / \mathrm{h}$ [95\% confidence interval 3.63-5.39] for a $70 \mathrm{~kg}$ individual) compared with that previously reported for healthy adults. Model-based simulations indicated similar variability in mean \pm standard deviation area under the curve from time zero to infinity of $1907 \pm 1552$ and $2175 \pm 1670 \mathrm{ng} \times \mathrm{h} / \mathrm{mL}$ in the $60-70 \mathrm{~kg}$ weight group, after $8 \mathrm{mg}$ fixed- or weight-based dosing, respectively.

Conclusion We describe the first PPK model for moxidectin in adults with S. stercoralis infection. Equivalent exposures after fixed-dose and weight-dependent dosing strategies support the use of a simple fixed-dose approach, particularly in large-scale treatment programs.
\end{abstract}

Trial Registration Registered at ClinicalTrials.gov (NCT04056325).

Cornelis Smit and Daniela Hofmann have shared first-authorship of this article.

\section{Jennifer Keiser}

jennifer.keiser@swisstph.ch

$\triangle$ Marc Pfister

marc.pfister@ukbb.ch

1 Pediatric Pharmacology and Pharmacometrics Research, University of Basel Children's Hospital (UKBB), Spitalstrasse 33, 4056 Basel, Switzerland

2 Department of Medical Parasitology and Infection Biology, Swiss Tropical and Public Health Institute, University of Basel, Socinstrasse 57, 4051 Basel, Switzerland

3 University of Basel, Basel, Switzerland

4 Lao Tropical and Public Health Institute, Vientiane, Lao People's Democratic Republic

5 Certara, Princeton, NJ, USA

\section{Introduction}

Strongyloides stercoralis belongs to the soil-transmitted helminths (STHs) and is the most neglected among the clinically relevant helminths. Nevertheless, with a worldwide prevalence of about 100 million infections, mainly in lowresource countries, strongyloidiasis is responsible for significant morbidity [1-3]. Today, the core strategy in combating helminth infections is chemotherapy combined with health education programs [4]. Anthelminthic treatment options are sparse; for strongyloidiasis, ivermectin is the only highly effective drug $[2,5]$. However, among new candidates in the pipeline, moxidectin has increased the prospects for a new alternative.

Moxidectin is from the same macrocyclic lactone family as ivermectin and therefore also exerts its action by binding to the glutamate-gated chloride channels and $\gamma$-aminobutyric 


\section{Key Points}

We characterized the population pharmacokinetics of moxidectin using data from Strongyloides stercoralisinfected adult patients treated with moxidectin 2-12 mg.

While body weight influences moxidectin clearance, we found high clearance in S. stercoralis-infected individuals compared with that reported for healthy subjects.

The pharmacokinetic profile of moxidectin shows substantial random variability, where both fixed- and weight-dependent dose strategies result in equivalent variable exposures across body weight. This supports the use of a fixed-dose strategy in large-scale programs for treating strongyloidiasis.

acid receptors, resulting in increased permeability and, ultimately, muscle paralysis [6]. In 2018, moxidectin was approved for use in humans aged at least 12 years for the treatment of onchocerciasis, having demonstrated superiority to ivermectin in phase II and III trials [7, 8]. Due to its good safety and tolerability profile, moxidectin is also currently being evaluated against lymphatic filariasis, strongyloidiasis, other STH infections and scabies. In treating these diseases, moxidectin may offer some advantages over ivermectin. For example, moxidectin is currently registered for onchocerciasis with a fixed-dose regimen, that is in contrast to ivermectin where doses need to be adjusted for body weight. Fixed-dose regimens facilitate large-scale treatment programs and community-based treatments by non-medical personnel [4]. In addition, the long elimination half-life of moxidectin observed in pharmacokinetic (PK) studies in animals and healthy individuals may be beneficial in controlling prolonged parasitic activity [9], which would be of particular value in the treatment of $S$. stercoralis that can persist in the human body during long periods of time.

A recently concluded phase IIa dose-finding study investigating moxidectin for the treatment of strongyloidiasis showed cure rates similar to those for ivermectin and indicated moxidectin was well tolerated [10]. It was concluded that the $8 \mathrm{mg}$ dose currently registered for use in onchocerciasis also appears suitable for treating strongyloidiasis. The maximum cure rates of around $80-90 \%$ observed in the study still call for improvement as the unique ability of S. stercoralis to replicate in the host (autoinfection) means that complete cure rates are sought for ultimate treatment success.
Despite the emerging knowledge on moxidectin in the treatment of STH, the population PK (PPK) profile of moxidectin required for regulatory approval remains to be investigated with regard to target populations outside of onchocerciasis. To date, only a limited number of PK analyses are publicly available [9, 11-14]. These studies, all conducted in healthy subjects with only a descriptive (non-compartmental) PK analysis, have provided us with important insights into the pharmacological profile of moxidectin. First, moxidectin has a relatively large apparent volume of distribution $\left(V_{\mathrm{d}} / F\right)$, likely due to its lipophilic properties [9]. Reported $V_{\mathrm{d}} / F$ values are around $3000 \mathrm{~L}$ in young, normal-weight healthy individuals, resulting in long elimination half-lives ranging from 20 to 48 days. This might be the result of both high tissue penetration, especially into fatty tissues, as has been demonstrated in several veterinary studies $[15,16]$, and the relatively low affinity of moxidectin for the drug transporter P-glycoprotein [17]. Second, moxidectin exposure is highly variable, with coefficients of variation (\%CV) of up to $50 \%$ in healthy subjects, which were treated with a single fixed dose [11-14]. Here, a PPK analysis is needed to provide more insight about the magnitude and possible sources of this variability in infected individuals. Moreover, it has repeatedly been shown that not only gastrointestinal nematode infections but also physiological abnormalities such as malnutrition or undernutrition can potentially affect the PK of a drug [18, 19], hence population PK studies in infected patients are required.

The objectives of this study were threefold: (1) to characterize the PPK of moxidectin in S. stercoralis-infected adults enrolled in the recently concluded phase IIa dosefinding study [10]; (2) to identify key covariate effects on PK parameters and quantify variability in PK parameters in these patients; (3) to perform model-based simulations to compare dosing strategies for $S$. stercoralis-infected adults for use in general, especially in strongyloidiasis, to maximize the efficacy of moxidectin while minimizing the complexity of dosing in large patient populations.

\section{Methodology}

\subsection{Study Design and Field Procedure}

The PK study was embedded in a phase IIa randomized, single-blinded, placebo-controlled, dose-escalation trial for the treatment of $S$. stercoralis-infected adults in Northern Laos (NamBak District). Enrolled S. stercoralis-infected adults were assigned by a computer-generated block randomization code to treatment arms of equal size, stratified by baseline infection intensity. In this PK study, single fixed doses of 2 , $4,6,8,10$, or $12 \mathrm{mg}$ of moxidectin were administered to 96 volunteer study participants. Moxidectin was administered 
as $2 \mathrm{mg}$ tablets provided by Medicines Development for Global Health. Before treatment administration, a local lunch (meat and noodle soup) was given. Capillary blood was collected in doublets by finger puncture using a $2.3 \mathrm{~mm}$ finger pricker and subsequent soaking using the volumetric microsample Mitra ${ }^{\circledR} 30 \mu \mathrm{L}$ (Neoteryx ${ }^{\circledR}$, Torrance, CA, USA) at different time points: prior to dosing, and 2, 4, 6, $7 \mathrm{~h}$, and $1,3,7$, and 28 days post-dose. The Mitra ${ }^{\circledR}$ sticks were allowed to dry and were then shipped to Basel, Switzerland, for analysis, after which the samples were analyzed within 1 month. For each participant, data on potential covariates such as age, height, weight, sex, baseline infection intensity (based on sextuplicate larvae counts per gram of feces), and information on co-infections (such as Trichuris trichiuria, Ascaris lumbricoides or hookworms) were recorded.

\subsection{Quantification of Moxidectin by Liquid Chromatography-Tandem Mass Spectrometry}

Moxidectin was analyzed by a previously validated liquid chromatography-tandem mass spectrometry (LC-MS/MS) method [20]. To account for the low-dosing groups and sampling over a long follow-up period, the calibration line was extended, compared with the published method. In brief, moxidectin was extracted from Mitra ${ }^{\circledR}$ with $300 \mu \mathrm{L}$ solution $(40 \mathrm{ng} / \mathrm{mL}$ moxidectin-d 3 in methanol:acetonitrile [4:1, $\mathrm{v} / \mathrm{v}]$ ) following $1 \mathrm{~h}$ of shaking and $1 \mathrm{~h}$ of sonication. The evaporated samples were reconstituted in $100 \mu \mathrm{L}$ methanol spiked with $1 \mathrm{mM}$ ammonium format and injected $(15 \mu \mathrm{L})$ into the LC-MS/MS system. Chromatographic separation was performed using a Luna C $8(2)$ column $(30 \times 2.0 \mathrm{~mm}$, $3 \mu \mathrm{m}$ particle size, $100 \AA$ ) and mobile phases of $0.05 \%$ formic acid in ultrapure water (mobile A) or methanol (mobile B), with a gradient program $(0-0.16 \mathrm{~min}, 80 \% \mathrm{~B}$; 3-4 min, $100 \% \mathrm{~B}$; $4.01-6 \mathrm{~min}, 80 \% \mathrm{~B}$ ) and a flow rate of $0.4 \mathrm{~mL} / \mathrm{min}$. The mass transitions 640.4 to $528.5 \mathrm{~m} / \mathrm{z}^{+}$and 643.4 to 531.5 $\mathrm{m} / \mathrm{z}^{+}$served to analyze moxidectin and the internal standard moxidectin-d3, respectively. Modification to the previously established method includes a $5 \mu \mathrm{L}$ larger injection, which allowed us to establish a lower limit of quantification (LLOQ) of $1.5 \mathrm{ng} / \mathrm{mL}$ instead of $2.5 \mathrm{ng} / \mathrm{mL}$. The assay was linear between $1.5 \mathrm{ng} / \mathrm{mL}$ and $250 \mathrm{ng} / \mathrm{mL}\left(R^{2}>0.99\right)$. The limit of detection was verified with a more than fivefold larger signal in the LLOQ samples compared with blank samples. The QC samples displayed an accuracy of between 89.3 and $107.5 \%$ and an imprecision of $<14.5 \%$, and as such were in accordance with regulatory guidelines. Accuracy and precision data are summarized in electronic supplementary Table S1. The extraction and measurement of $9.7 \%$ of samples was repeated and $96.8 \%$ thereof deviated $<15 \%$ from the initially evaluated concentration, thus fulfilling the criteria of accuracy.

\subsection{Population Pharmacokinetic Analysis}

Concentration-time data were fitted using non-linear mixedeffects modeling using the Stochastic Approximation Expectation Maximization (SAEM) algorithm in the Monolix 2019R2 software package (version 5.1.1) [21]. Data manipulation, statistical analysis, simulations, and visualization were performed using $\mathrm{R}$ version 4.0.2 and Rstudio version 1.3.959 [22]. Pre-dose concentrations were used to confirm no individuals were pretreated with moxidectin, but were not included in the PK analysis. A base PPK model was constructed by testing one-, two-, and three-compartment models with first-order absorption and linear elimination. Delayed absorption models were investigated with a lagtime parameter and a fixed or estimated number of transit compartment models [23]. Interindividual variability (IIV) on parameter estimates was assumed to be log-normally distributed, and residual variability was modeled using additive, proportional, or combined error structures.

Left-censored observations (below the LLOQ [BLQ]) were handled by an extension of the SAEM algorithm, as is default in Monolix, following a method comparable with the M3 method used within NONMEM [24]. Nested models were evaluated and compared based on the objective function value (OFV, or - 2 log likelihood [-2LL]) and nonnested models using the corrected Bayesian information criterion (BICc), both estimated by importance resampling and reported using Monolix. A decrease of 3.84 points in the $\mathrm{OFV}$, corresponding to $p<0.05$, or a decrease in BICc indicates a significantly better model. Other criteria for selection of the best model were goodness of fit (GOF), parameter precision (depicted by the relative standard error), overall model stability, and the conditional number.

Available covariates were weight, lean body weight (LBW, based on the Janmahasatian equation [25]), body mass index (BMI), age, and sex. Baseline infection intensity was investigated as a categorical covariate (light, moderate, heavy for larvae per gram [LPG] of $\geq 0.4$ to $1, \geq 1$ to 10 , or $\geq 10$, respectively). Co-infections were recorded at baseline as a combined variable with a value of 1 or 0 depending on whether any co-infection was observed. Utilizing the base PPK model, covariates were explored by plotting the individual parameters using random samples from the conditional distribution ( $n=7$ per individual) versus individual random-effect values, where, based on a visual inspection of these plots, covariates were entered into the model when showing a possible correlation. Continuous covariates were entered in the model according to Eq. 1:

$\log P_{i}=\log P_{p}+X \times \log \left(\frac{\mathrm{COV}_{i}}{\mathrm{COV}_{\text {standard }}}\right)$ 
where $P_{i}$ and $P_{p}$ represent individual and population parameter estimates, $\mathrm{COV}_{\mathrm{i}}$ represents the individual covariate value, $\mathrm{COV}_{\text {standard }}$ represents a standardized (e.g. $70 \mathrm{~kg}$ for weight) or median value for the covariate, and $X$ reflects the effect of $\mathrm{COV}$ on parameter $P$. Categorical covariates (with values of either 0 or 1 ) were tested using Eq. 2:

$\log P_{i}=\log P_{p}+Z_{\mathrm{COV}_{i}=1}$

where $P_{i}$ and $P_{p}$ represent individual and population parameter estimates and $Z_{\mathrm{COV}_{i}=1}$ is the effect of COV on parameter $P$ when the covariate has a value of 1 . Covariates were entered by assessment of the following criteria: a reduction of the OFV of more than 3.84 points, improvement of the GOF, a decrease in IIV of the population parameter, and clinical significance of the covariate effect. Covariates were included stepwise, with the covariate with the largest OFV reduction and biggest improvement in GOF included first. After covariate inclusion, correlation between random effects was visually assessed and included in the final model if present.

The final model was internally validated by predictioncorrected visual predictive checks (pcVPC). Additionally, model performance was assessed by assessment of normalized prediction distribution errors (NPDE). Model stability was assessed and $95 \%$ confidence intervals (CIs) were obtained by non-parametric bootstrap $(n=1000)$ using the Rsmlx R package.

Applying the final PPK model, terminal elimination halflife (in hours; $T_{1 / 2}{ }^{\alpha}$ for a one-compartment model and $T_{1 / 2}{ }^{\beta}$ for a two-compartment model) was calculated for a typical individual from the estimated PK parameters using the PKconverter R package [26].

\subsection{Model-Based Simulations to Inform Dosing Strategy}

To investigate the need for a weight-adjusted dose regimen over a fixed-dose regimen, we performed dose simulations in 5000 virtual S. stercoralis-infected subjects with a randomly assigned weight and age based on the range found in the original dataset. Simulations were performed using the final PK model with IIV. Two single-dose regimens were simulated: (1) a single-dose based on weight using the relationship between clearance and weight as identified in the PK model; and (2) the previously suggested single fixed dose of $8 \mathrm{mg}$ [10]. For the weight-based dose regimen (1), we determined a weight-based dose relative to an $8 \mathrm{mg}$ dose administered to an individual weighting $56 \mathrm{~kg}$, which is the median weight of participants in our dataset. Using the relationship between weight and CL as incorporated in the final PPK model, dose was calculated using the following equation (Eq. 3):
DOSE $=\frac{8}{\mathrm{DW}_{56 \mathrm{~kg}}} \times \mathrm{DW}$

$\mathrm{DW}=70 \times\left(\frac{\mathrm{WT}}{70}\right)^{0.75}$

where DOSE is the dose in mg, DW represents a 'dose weight' in $\mathrm{kg}$, with $\mathrm{DW}_{56 \mathrm{~kg}}$ being the $\mathrm{DW}$ of a $56 \mathrm{~kg}$ person, calculated using Eq. 4, where WT is the body weight in kilograms.

For each subject, area under the curve from time zero to infinity $\left(\mathrm{AUC}_{\infty}\right)$ was calculated using Eq. 5:

$\mathrm{AUC}_{\infty}=\frac{\mathrm{DOSE}}{\mathrm{CL} / F}$

where $\mathrm{AUC}_{\infty}$ is the exposure from time zero to infinity in $\mathrm{ng} \times \mathrm{h} / \mathrm{mL}$, DOSE is the dosage in micrograms, and CL/F is the apparent clearance in liters/hour. Simulations were performed in $\mathrm{R}$ using the simulx package (part of the mlxR package).

\section{Results}

\subsection{Pharmacokinetic Data}

Between November 2019 and March 2020, a total of 96 individuals were enrolled in the PK study of the phase IIa trial. Individuals were equally assigned to six dose groups ranging from moxidectin 2-12 mg, with 15-21 individuals in each arm. No moxidectin was detected in any pre-dose sample. Baseline characteristics of the study population are shown in Table 1. In total, 762 moxidectin samples were collected. Compared with the planned sampling scheme, six samples were missed due to the unavailability of the study participant. Of the 762 collected PK samples, 158 (20.7\%) were BLQ. Electronic Supplementary Fig. S1 shows the observed moxidectin concentrations stratified by dose group.

\subsection{Population Pharmacokinetic Analysis}

All 762 PK samples were used to develop a PPK model. A two-compartment model with a proportional error model was found to best describe the data. Delayed absorption was included in the model using a depot compartment with lag time ( $T_{\text {lag }} ; \Delta \mathrm{OFV}-158.1$ points compared with the model without lag time). This model outperformed a model with an estimated number of transit compartments $(\Delta \mathrm{OFV}-116.1$ points compared with a model without a delayed absorption). We were able to estimate IIV with reasonable precision for the absorption rate constant $\left(k_{\mathrm{a}}\right), \mathrm{CL} / F, \mathrm{~V}_{\mathrm{d}} / F$ of the central and peripheral compartments $\left(V_{1} / F\right.$ and $\left.V_{2} / F\right)$, and 
Table 1 Baseline characteristics

\begin{tabular}{ll}
\hline Characteristic & $\begin{array}{l}\text { Individuals in the pharma- } \\
\text { cokinetic dataset }[n=96]\end{array}$ \\
\hline Age, years & $45.0(39.0-53.3)[22-65]$ \\
Height, cm & $158(152-163)[137-172]$ \\
Total body weight, $\mathrm{kg}$ & $56.2(51.1-61.9)[36.2-82.6]$ \\
Body mass index, kg/m ${ }^{2}$ & $22.2(20.9-24.3)[17.0-32.3]$ \\
Sex $[n(\%)]$ & Male: $59(61)$ \\
Infection intensity at baseline $[n(\%)]$ & Lemale: $37(39)$ \\
& Light: $16(16.7)$ \\
Doderate: $38(39.6)$ \\
Heavy: $42(43.6)$ \\
2 mg: $15(15.6)$ \\
4 mg: $16(16.7)$ \\
6 mg: $14(14.6)$ \\
8 mg: $21(21.9)$ \\
$10 \mathrm{mg}: 15(15.6)$ \\
$12 \mathrm{mg}: 15(15.6)$
\end{tabular}

Data are expressed as median (interquartile range) [full range] unless specified otherwise

apparent intercompartmental clearance $(Q / F)$. Removal of any of these IIV parameters from the model resulted in a significant increase in OFV. For $T_{\text {lag }}$, we fixed the IIV parameter at 0.1 since there was a large correlation between IIV on $T_{\text {lag }}$ and $k_{\mathrm{a}}$, but removing IIV on $T_{\text {lag }}$ resulted in worsening of GOF and a significant increase in OFV $(\Delta \mathrm{OFV}+61.7$ points compared with the model with IIV on $T_{\text {lag }}$ ). When this value was fixed at 0.1 , both the correlation and conditional number decreased without losing GOF with a similar OFV. As a sensitivity analysis, we checked other values for fixing IIV on $T_{\text {lag }}$, namely $0.05,0.2$, and 0.5 , but these models resulted in a higher OFV $(\Delta \mathrm{OFV}+6.0,+3.5$, and +28.3 , respectively, compared with the model with IIV $T_{\text {lag }}$ fixed at 0.1 ).

For the covariate analysis, we identified age, weight, and sex as possible covariates based on parameter versus covariate plots (Electronic Supplementary Fig. S2). We implemented the effect using an allometric model, resulting in an improvement in GOF and a significant reduction in OFV $(\triangle \mathrm{OFV}-15$ points compared with the structural model without covariates). In the next step, we included age as a covariate for $V_{1} / F$, resulting in a reduction in OFV and a better description of peak concentrations in GOF plots $(\triangle \mathrm{OFV}-8.1$ points compared with the model without age on $\left.V_{1} / F\right)$. The age effect was included using a power equation with an estimated exponent of $-0.422(95 \% \mathrm{CI}-0.674$ to -0.197$)$, indicating a reduction of $V_{1}$ from $199 \mathrm{~L}(95 \%$ CI $162-250 \mathrm{~L})$ to $116 \mathrm{~L}(95 \% \mathrm{CI} 105-126 \mathrm{~L})$ for a typical individual with a weight of $70 \mathrm{~kg}$ and aged 18 and 65 years, respectively. No effect of age could be identified for any of the other PK parameters. Lastly, sex as a covariate for $V_{2} / F$ was found to be statistically significant $(\Delta \mathrm{OFV}-9.8$ points), but given the absence of a clear improvement in GOF, we decided to not include this covariate in the model. None of the remaining covariates, including baseline infection intensity or the presence of a co-infection, showed any correlation with any random-effects parameters, indicating that the covariate analysis was complete. In addition, no correlation between random effects was present. The final PPK model showed good description of the observed and BLQ data based on the pcVPC (Fig. 1), GOF plots (Electronic Supplementary Fig. S3), and distribution of the NPDE (Electronic Supplementary Fig. S4). The bootstrap analysis indicates good model stability (Table 2).

The parameters of the final model are shown in Table 2. Using the obtained parameters from the final PPK model, we calculated a $T_{1 / 2}$ of $278 \mathrm{~h}$ for an individual with a weight of $70 \mathrm{~kg}$ and aged 44.3 years.

\subsection{Model-Based Simulations to Inform Dosing Strategy}

To compare the originally proposed $8 \mathrm{mg}$ fixed-dose strategy [10] with a weight-based dosing strategy, we performed dose simulations using both strategies. For each dosing strategy, the quantitative results of the simulations performed in 5000 virtual subjects with randomly assigned weight between 30 and $90 \mathrm{~kg}$ and aged between 18 and 65 years are shown in electronic supplementary Tables S2 and S3. The mean \pm standard deviation $\mathrm{AUC}_{\infty}$ in the $60-70 \mathrm{~kg}$ group is similar for both dose groups, at $1907 \pm 1552$ and $2175 \pm 1670 \mathrm{ng}$ $\times \mathrm{h} / \mathrm{mL}$ for an $8 \mathrm{mg}$ fixed dose and weight-based dosing, respectively. Figure 2 shows the distribution of $\mathrm{AUC}_{\infty}$ split for weight group, indicating the high variability in exposure, with similar results obtained for both the fixed-dose and weight-based dosing strategies. The exposure remains fairly constant across different weights regardless of the dosing strategy. The distribution of the obtained peak concentrations is shown in Electronic Supplementary Fig. S5, indicating alike results as found for exposure: While peak concentrations are similar for both dosing strategies, they appear to differ in the highest and lowest weight groups $(<$ 40 or $>70 \mathrm{~kg}$ ).

\section{Discussion}

In this study, we have successfully developed a PPK model of moxidectin using data from a large cohort of $S$. stercoralis-infected patients. To our knowledge, this is the first PPK study for moxidectin in humans and the first PK analysis of this drug in patients, as only a few descriptive studies in healthy subjects have been conducted to date $[9$, 

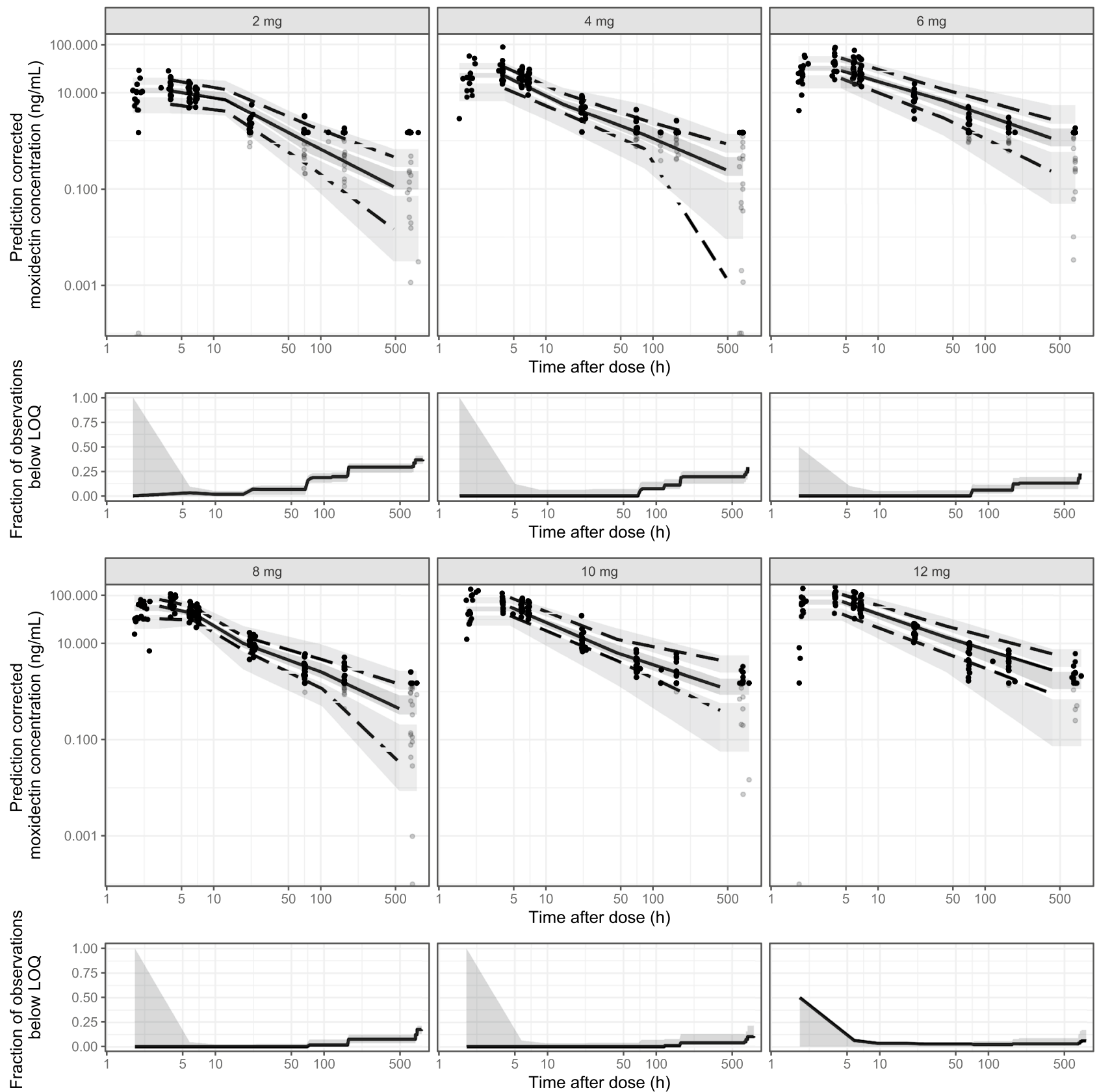

Fig. 1 Prediction-corrected visual predictive check for the final pharmacokinetic model, split by dose group. Upper panels: lines represent the 5th (dashed), 50th (solid), and 95th (dashed) percentiles of the prediction-corrected observed data. The shaded areas indicate the $90 \%$ confidence intervals of the 5th, 50th, and 95th percentiles of the simulated data ( $n=500$ datasets). The black and grey circles corre-

11-14]. Pharmacometric modeling and simulation studies are urgently needed to inform drug dosing for future clinical trials or clinical use of moxidectin in several disease areas, such as STH infections or scabies, similar to recent efforts involving ivermectin and tribendimidine [27-29]. spond to the prediction-corrected observed values and simulated values for observations below the LLOQ, respectively. Lower panels: the solid line represents the fraction of observations below the LLOQ, with the area indicating the $95 \%$ prediction interval of the fraction below the LLOQ based on the simulations $(n=500)$. LLOQ lower limit of quantification, $L O Q$ limit of quantification
Our model-based simulations show that adult patients with a weight between 60 and $70 \mathrm{~kg}$ who receive a fixed dose of moxidectin $8 \mathrm{mg}$ are expected to have a mean exposure of $1907 \pm 1552 \mathrm{ng} \times \mathrm{h} / \mathrm{mL}$. This is lower than what has been observed in studies with healthy subjects receiving the same 
Table 2 Population pharmacokinetic parameters of the final population pharmacokinetic model

\begin{tabular}{|c|c|c|}
\hline Parameter & Estimate (\%RSE) & $\begin{array}{l}\text { Bootstrap estimate } \\
{[n=1000](95 \% \mathrm{CI})}\end{array}$ \\
\hline \multicolumn{3}{|l|}{ Population parameters } \\
\hline$T_{\text {lag }}[\mathrm{h}]$ & $1.64(1.6)$ & $1.63(1.56-1.70)$ \\
\hline$K_{\mathrm{a}}[/ \mathrm{h}]$ & $3.38(11)$ & $3.35(2.53-4.44)$ \\
\hline $\mathrm{CL} / F_{\text {pop }}[\mathrm{L} / \mathrm{h}]$ & $4.47(7.6)$ & $4.47(3.63-5.39)$ \\
\hline$V_{1} / F_{\text {pop }}[\mathrm{L}]$ & $136(3.4)$ & $136(126-146)$ \\
\hline$Q / F_{\text {pop }}[\mathrm{L} / \mathrm{h}]$ & $10.0(4.5)$ & $10.0(9.10-11.0)$ \\
\hline$V_{2} / F_{\text {pop }}[\mathrm{L}]$ & $1172(11)$ & $1169(875-1523)$ \\
\hline \multicolumn{3}{|l|}{ Covariate effects ${ }^{\mathrm{a}}$} \\
\hline WT on CL/F & 0.75 FIX & - \\
\hline WT on $V_{1} / F$ & 1 FIX & - \\
\hline AGE on $V_{1} / F$ & $-0.42(30)$ & $\begin{array}{l}-0.43(-0.67 \text { to }- \\
0.20)\end{array}$ \\
\hline WT on $Q / F$ & 0.75 FIX & - \\
\hline WT on $V_{2} / F$ & 1 FIX & \\
\hline \multicolumn{3}{|c|}{ Interindividual variability } \\
\hline$\omega T_{\text {lag }}$ & 0.1 FIX & 0.1 FIX \\
\hline$\omega K_{\mathrm{a}}$ & $0.672(14)$ & $0.604(0.355-0.850)$ \\
\hline$\omega \mathrm{CL} / F$ & $0.625(8.6)$ & $0.627(0.519-0.724)$ \\
\hline$\omega V_{1} / F$ & $0.297(9.2)$ & $0.291(0.220-0.379)$ \\
\hline$\omega Q / F$ & $0.343(11)$ & $0.344(0.236-0.445)$ \\
\hline$\omega V_{2} / F$ & $0.779(11)$ & $0.743(0.436-0.976)$ \\
\hline \multicolumn{3}{|l|}{ Residual variability } \\
\hline Proportional error ${ }^{\mathrm{b}}$ & $0.165(4.9)$ & $0.169(0.144-0.199)$ \\
\hline
\end{tabular}

$A G E$ age in years, $C I$ confidence interval, $C L / F$ apparent clearance, $C L / F_{\text {pop }}$ population value for $\mathrm{CL} / \mathrm{F}$ for a person with a body weight of $70 \mathrm{~kg}, K_{a}$ absorption constant, $Q / F$ apparent intercompartmental clearance, $Q / F_{\text {pop }}$ population value for $\mathrm{Q} / \mathrm{F}$ for a person with a body weight of $70 \mathrm{~kg}, T_{\text {lag }}$ absorption lag time, $V_{l} / F$ apparent volume of distribution of the central compartment, $V_{l} / F_{\text {pop }}$ population value for $V_{1} / F$ for a person with a body weight of $70 \mathrm{~kg}$ and aged 44.3 years, $V 2 / F$ apparent volume of distribution of the peripheral compartment, $V 2 / F_{\text {pop }}$ population value for $V_{1} / F$ for a person with a body weight of $70 \mathrm{~kg}, W T$ total body weight in kilograms, $\omega$ standard deviation of the interindividual variability parameter, $\% R S E$ percentage relative standard error

${ }^{\mathrm{a}}$ Covariate parameter relationships: $\mathrm{CL} / F=\mathrm{CL} / F_{\text {pop }} \times(\mathrm{WT} / 70)^{0.75}$. $V_{1} / F=V_{1} / F_{\text {pop }} \times(\mathrm{WT} / 70) \times(\mathrm{AGE} / 44.3)^{-0.42} \cdot \stackrel{Q}{Q} / F=Q / F_{\text {pop }} \times$ $(\mathrm{WT} / 70)^{0.75} \cdot V_{2} / F=V_{2} / F_{\text {pop }} \times(\mathrm{WT} / 70)$

${ }^{\mathrm{b}}$ Residual proportional error shown as the standard deviation

dose $[12,14]$, where a mean AUC of approximately 4500 $\mathrm{ng} \times \mathrm{h} / \mathrm{mL}$ was reported. This is the result of high clearance in patients, i.e. $4.47 \mathrm{~L} / \mathrm{h}$ (95\% CI 3.63-5.39), markedly higher than what is reported in healthy subjects (varying between 1.9 and $3.5 \mathrm{~L} / \mathrm{h}$ ) $[9,11-14]$. This also results in a shorter calculated $T_{1 / 2}$ of $278 \mathrm{~h}$ for patients, whereas values of 528-1139 h have been reported for healthy subjects [9, 11-14]. These results indicate that infection could influence the PK of moxidectin, resulting in a decrease in total moxidectin exposure. This finding is in line with what has been observed in veterinary studies [30], but is in contrast to findings for ivermectin, a related macrocyclic lactone, where infection with Onchocerca volvulus did not influence the PK [31]. Unfortunately, we could not relate the baseline infection intensity to any PPK parameter. One limitation in this respect is that healthy subjects were not included due to the nature of this clinical study. Therefore, any firm conclusions on the influence of illness on moxidectin PK cannot be made based on the current study alone. In addition, several other factors could influence the PK of moxidectin, such as study design, ethnicity, patient characteristics, drug formulation, and food status. All studies in healthy subjects analyzed the data using a non-compartmental analysis. Assuming moxidectin follows a bi-exponential elimination, the assessment of clearance in such a noncompartmental analyses could have been biased. However, when using an appropriate sampling design, this bias is usually limited, even for drugs with a two-compartmental PK profile [32]. In addition, investigations with healthy subjects were almost exclusively conducted in European study sites, where participants were predominantly Caucasian, in contrast to our study [9, 11-14]. It is currently unknown if ethnic differences influence moxidectin PK. Regarding patient characteristics, individuals in our study had a lower BMI (median 22.2) compared with healthy subjects (median BMI around 24-25). Due to the high lipophilicity of moxidectin, a higher BMI in healthy individuals could lead to increased tissue accumulation, with a decrease in clearance. However, drug lipophilicity alone does not immediately translate to an increased clearance, as, for example, has been shown for the high lipophilic drug diazepam in obese individuals where increased accumulation but a similar clearance was observed [33]. A follow-up study including obese participants would be necessary to further investigate this alternative hypothesis. Drug formulation (oral solution vs. tablet) and food status are also known modulators of moxidectin PK [9, 11]. In this study, moxidectin was administered as the commercially available tablet after consumption of food and as such highly comparable with most published studies in healthy subjects $[11,12,14]$, thus making it unlikely that these factors might explain the observed differences.

We observed considerable unexplained variability for only a small part, described by available covariates. The resulting variation in expected exposures observed in modelbased simulations are in the same order of magnitude as has been reported in the healthy subjects. For example, KorthBradley et al. reported an $\mathrm{AUC}_{\infty}$ of $4403 \pm 2360 \mathrm{ng} \times \mathrm{h} / \mathrm{mL}$ after a single dose of moxidectin $10 \mathrm{mg}$ administered to 29 healthy individuals [11]. This translates to a $\% \mathrm{CV}$ of around $54 \%$, similar to what we observed in our simulated data after an $8 \mathrm{mg}$ fixed dose. Importantly, given the finding that most variability in moxidectin exposure appears to be random, our results support the previously proposed fixed-dose strategy 
Fig. $2 \mathrm{AUC}_{\infty}$ values obtained following simulations with either a fixed-dose (black boxplots) or weight-based (grey boxplots) dosing strategy, based on the pharmacokinetic model in individuals with varying age and body weight $(n=5000$ per dosing strategy). Each boxplot represents the median and interquartile range for each weight group. $A U C_{\infty}$ area under the curve from time zero to infinity

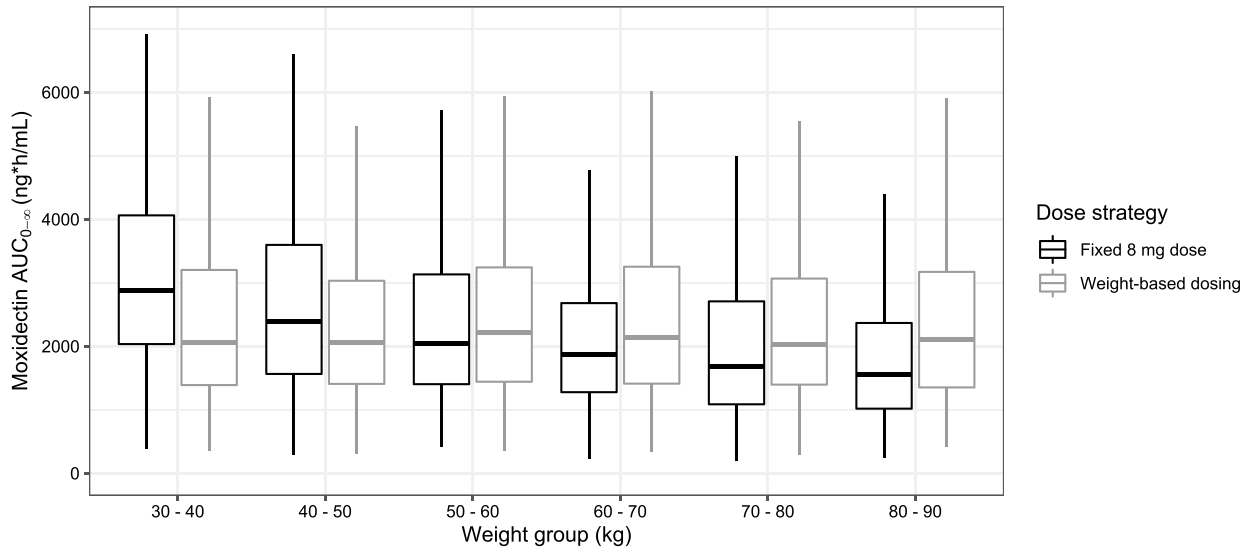

[10]. We have illustrated that applying a weight-dependent dosing strategy did not reduce variability in exposure and hence has no clear benefit over a fixed-dose strategy. This means that, in contrast to ivermectin, the currently only efficacious treatment option against $S$. stercoralis, moxidectin dose, does not have to be adjusted according to weight. In theory, there might be other covariates that are capable of predicting the PK of moxidectin. However, we have to consider that moxidectin will be mainly applied in low-resource income settings during mass drug administrations, where dose individualization is not practical. Studies that combine PK information with outcome data in order to establish an exposure-response relationship, to determine target exposures and, if needed, to further fine tune the current dose recommendations are ongoing.

Some limitations apply to our study. Firstly, our study only included adult patients from Southeast Asia with relatively normal weight and age ranges for that location. Although we have included both age and weight as covariates in the PK model, we have to be cautious in extrapolating our results to cases on the far end of each spectrum, such as children or obese individuals. For such special populations it is well known that a simple extrapolation from (normalweight) adults might not be appropriate [34, 35]. Furthermore, we only had one individual in the study with a weight of $<40 \mathrm{~kg}$. This could limit the applicability of our model and simulations for this group of individuals. In addition, caution is necessary when extrapolating our results to populations in other areas of the world. As such, more research is needed to validate or extend the PK model to other populations than those studied here. Secondly, although we were able to estimate the population value for $T_{\text {lag }}$ and $K_{\mathrm{a}}$ with reasonable precision, the inability to estimate IIV on the $T_{\text {lag }}$ parameter might reflect the sparsity of our data around the absorption phase, with the first sample drawn at $2 \mathrm{~h}$ after the moxidectin dose. Therefore, our findings regarding the absorption profile of moxidectin should be considered with some caution. Thirdly, in this study, we did not measure renal and liver function; however, no significant influence of these covariates on the PK of moxidectin is expected, since it is known that moxidectin is predominantly cleared via a non-renal route with only minimal hepatic metabolism [36]. Lastly, since we used a capillary micro blood sampling methodology to quantify moxidectin concentrations, a relatively large part of the moxidectin concentration in the later elimination phase was below the assay's LOQ. Nonetheless, these BLQ measurements could be retained in the dataset and analyzed by using a method similar to the widely accepted M3 method that was developed for NONMEM, where for each BLQ observation, the likelihood of this value actually being BLQ is estimated [24]. Hence, we could limit the loss of information due to a relatively large portion of BLQ measurements.

\section{Conclusions}

We have successfully characterized the PPK of moxidectin in S. stercoralis-infected individuals. To our knowledge, this is the first study to report the PPK of moxidectin in a human population, and, additionally, the first study to report the PPK of moxidectin in adults with a helminth infection. We found high clearance in S. stercoralis-infected participants compared with what is reported in healthy subjects, indicating a possible influence of infection on moxidectin PK. Furthermore, the PK profile of moxidectin is marked by unexplained variability in drug exposure, which is equivalent after a fixed dose and weight-based dosing. As such, there is no clear rationale to support weight-based dose adjustments over a simple fixed-dose strategy in the application of moxidectin in S. stercoralis infections.

Supplementary Information The online version contains supplementary material available at https://doi.org/10.1007/s40262-021-01048-4.

Acknowledgements The authors thank Prof. Dr. John. N. van den Anker and Dr. Mark D. Sullivan for critically reviewing this 
manuscript. The moxidectin tablets were manufactured and kindly provided by Medicine Development for Global Health.

\section{Declarations}

Funding Open Access funding provided by Universität Basel (Universitätsbibliothek Basel). Marc Pfister and Cornelis Smit are financially supported by the Eckenstein-Geigy Foundation in Basel, Switzerland. Jennifer Keiser and Daniela Hofmann are financially supported by the Adjuvare Foundation and the Swiss National Science Foundation (No. IZJFZ3_185646).

Conflicts of interest/Competing interests Marc Pfister has part-time employment with the consulting company Certara LP (USA). Cornelis Smit, Daniela Hofmann, Somphou Sayasone, and Jennifer Keiser declare no competing interests for this work.

Ethics approval Ethical clearance was obtained from the National Ethics Committee for Health Research on 12 September 2019 in Laos (reference no. 082-NECHR) and the Ethics Committee of Northwestern and Central Switzerland on 15 July 2019 (no. 2019-00558). This study was conducted in full accordance with the Declaration of Helsinki and International Conference on Harmonization Guidelines in Good Clinical Practice, and is registered at ClinicalTrials.gov (identifier: NCT04056325).

Consent to participate Written informed consent was obtained from all participants.

\section{Consent for publication Not applicable.}

Availability of data and material The study protocol for this publication is available on ClinicalTrials.gov (NCT04056325). Individual de-identified participant data that underlie the results reported in this article are available upon request, beginning directly after publication and ending 1 year after. Access will be granted to researchers who provide a scientifically sound proposal. Requests should be directed to Jennifer Keiser (jennifer.keiser@swisstph.ch). Data requestors will be required to sign a data access agreement prior to access being granted.

Code availability The MLXTRAN code for the final PK model is provided in the Electronic Supplementary File.

Open Access This article is licensed under a Creative Commons Attribution-NonCommercial 4.0 International License, which permits any non-commercial use, sharing, adaptation, distribution and reproduction in any medium or format, as long as you give appropriate credit to the original author(s) and the source, provide a link to the Creative Commons licence, and indicate if changes were made. The images or other third party material in this article are included in the article's Creative Commons licence, unless indicated otherwise in a credit line to the material. If material is not included in the article's Creative Commons licence and your intended use is not permitted by statutory regulation or exceeds the permitted use, you will need to obtain permission directly from the copyright holder. To view a copy of this licence, visit http://creativecommons.org/licenses/by-nc/4.0/.

\section{References}

1. Schär F, Trostdorf U, Giardina F, Khieu V, Muth S, Marti H, et al. Strongyloides stercoralis: Global Distribution and Risk Factors. PLoS Negl Trop Dis. 2013;7:e2288.
2. Krolewiecki AJ, Lammie P, Jacobson J, Gabrielli A-F, Levecke B, Socias E, et al. A public health response against Strongyloides stercoralis: time to look at soil-transmitted helminthiasis in full. PLoS Negl Trop Dis. 2013;7:e2165.

3. Olsen A, van Lieshout L, Marti H, Polderman T, Polman K, Steinmann P, et al. Strongyloidiasis-the most neglected of the neglected tropical diseases? Trans R Soc Trop Med Hyg. 2009;103:967-72.

4. Gabrielli A-F, Montresor A, Chitsulo L, Engels D, Savioli L. Preventive chemotherapy in human helminthiasis: theoretical and operational aspects. Trans R Soc Trop Med Hyg. 2011;105:683-93.

5. Henriquez-Camacho C, Gotuzzo E, Echevarria J, White AC, Terashima A, Samalvides F, et al. Ivermectin versus albendazole or thiabendazole for Strongyloides stercoralis infection. Cochrane database Syst Rev. 2016;1:CD007745.

6. Shoop WL, Mrozik H, Fisher MH. Structure and activity of avermectins and milbemycins in animal health. Vet Parasitol. 1995;59:139-56.

7. Opoku NO, Bakajika DK, Kanza EM, Howard H, Mambandu GL, Nyathirombo A, et al. Single dose moxidectin versus ivermectin for Onchocerca volvulus infection in Ghana, Liberia, and the Democratic Republic of the Congo: a randomised, controlled, double-blind phase 3 trial. Lancet. 2018;392:1207-16.

8. Awadzi K, Opoku NO, Attah SK, Lazdins-Helds J, Kuesel AC. A randomized, single-ascending-dose, ivermectin-controlled, double-blind study of moxidectin in Onchocerca volvulus infection. PLoS Negl Trop Dis. 2014;8:e953.

9. Cotreau MM, Warren S, Ryan JL, Fleckenstein L, Vanapalli SR, Brown KR, et al. The antiparasitic moxidectin: Safety, tolerability, and pharmacokinetics in humans. J Clin Pharmacol. 2003;43:1108-15.

10. Hofmann D, Sayasone S, Sengngam K, Chongvilay B, Hattendorf J, Keiser J. Efficacy and safety of ascending doses of moxidectin against Strongyloides stercoralis infections in adults: a randomised, parallel-group, single-blinded, placebo-controlled, dose-ranging, phase 2a trial. Lancet Infect Dis. 2021. https://doi. org/10.1016/S1473-3099(20)30691-5 (Epub 30 Mar 2021).

11. Korth-Bradley JM, Parks V, Patat A, Matschke K, Mayer P, Fleckenstein L. Relative bioavailability of liquid and tablet formulations of the antiparasitic moxidectin. Clin Pharmacol Drug Dev. 2012;1:32-7.

12. Korth-Bradley JM, Parks V, Wagner F, Chalon S, Gourley I, Matschke K, et al. Effect of moxidectin on CYP3A4 activity as evaluated by oral midazolam pharmacokinetics in healthy subjects. Clin Pharmacol Drug Dev. 2014;3:151-7.

13. Kinrade SA, Mason JW, Sanabria CR, Rayner CR, Bullock JM, Stanworth SH, et al. Evaluation of the cardiac safety of longacting endectocide moxidectin in a randomized concentration-QT study. Clin Transl Sci. 2018;11:582-9.

14. Korth-Bradley JM, Parks V, Chalon S, Gourley I, Matschke K, Gossart S, et al. Excretion of moxidectin into breast milk and pharmacokinetics in healthy lactating women. Antimicrob Agents Chemother. 2011;55:5200-4.

15. Afzal J, Burke AB, Batten PL, DeLay RL, Miller P. Moxidectin: metabolic fate and blood pharmacokinetics of 14C-labeled moxidectin in horses. J Agric Food Chem. 1997;45:3627-33.

16. Zulalian J, Stout SJ, DaCunha AR, Garces T, Miller P. Absorption, tissue distribution, metabolism, and excretion of moxidectin in cattle. J Agric Food Chem. 1994;42:381-7.

17. Lespine A, Martin S, Dupuy J, Roulet A, Pineau T, Orlowski $\mathrm{S}$, et al. Interaction of macrocyclic lactones with P-glycoprotein: Structure-affinity relationship. Eur J Pharm Sci. 2007;30:84-94.

18. Oshikoya KA, Sammons HM, Choonara I. A systematic review of pharmacokinetics studies in children with protein-energy malnutrition. Eur J Clin Pharmacol. 2010;66:1025-35. 
19. Geary TG, Woo K, McCarthy JS, Mackenzie CD, Horton J, Prichard RK, et al. Unresolved issues in anthelmintic pharmacology for helminthiases of humans. Int J Parasitol. 2010;40:1-13.

20. Hofmann D, Sayasone S, Keiser J. Development and validation of an LC-MS/MS method for the quantification of the anthelmintic drug moxidectin in a volumetric absorptive microsample, blood, and plasma: application to a pharmacokinetic study of adults infected with $S$. stercoralis. J Chromatogr B Anal Technol Biomed Life Sci. 2021. https://doi.org/10.1016/j.jchromb.2021. 122556 (Epub 1 Mar 2021).

21. Lixoft SAS. Monolix suite version 2019R2. 2019. https://www. lixoft.com/products/monolix/.

22. R Core Team. R: A language and environment for statistical computing. 2019. https://www.r-project.org/.

23. Savic RM, Jonker DM, Kerbusch T, Karlsson MO. Implementation of a transit compartment model for describing drug absorption in pharmacokinetic studies. J Pharmacokinet Pharmacodyn. 2007;34:711-26.

24. Beal SL. Ways to fit a PK model with some data below the quantification limit. J Pharmacokinet Pharmacodyn. 2001;28:481-504.

25. Janmahasatian S, Duffull SB, Ash S, Ward LC, Byrne NM, Green B. Quantification of lean bodyweight. Clin Pharmacokinet. 2005;44:1051-65.

26. Cho H, Lee E-K. PKconverter: R package to convert the pharmacokinetic parameters. Transl Clin Pharmacol. 2019;27:73-9.

27. Brussee JM, Schulz JD, Coulibaly JT, Keiser J, Pfister M. Ivermectin dosing strategy to achieve equivalent exposure coverage in children and adults. Clin Pharmacol Ther. 2019;106:661-7.

28. Brussee JM, Neodo A, Schulz JD, Coulibaly JT, Pfister M, Keiser J. Pharmacometric analysis of tribendimidine monoand combination therapies to achieve high cure rates in patients with hookworm infections. Antimicrob Agents Chemother. 2021;65(2):e00714-e720.
29. Brussee JM, Hiroshige N, Neodo A, Coulibaly JT, Pfister M, Keiser J. Population pharmacokinetics and exposure-response analysis of tribendimidine to improve treatment for children with hookworm infection. Antimicrob Agents Chemother. 2021;65(2):e01778-e1820.

30. Lespine A, Sutra JF, Dupuy J, Alvinerie M, Aumont G. The influence of parasitism on the pharmacokinetics of moxidectin in lambs. Parasitol Res. 2004;93:121-6.

31. Baraka OZ, Mahmoud BM, Marschke CK, Geary TG, Homeida MMA, Williams JF. Ivermectin distribution in the plasma and tissues of patients infected with Onchocerca volvulus. Eur J Clin Pharmacol. 1996;50:407-10.

32. Hughes JH, Upton RN, Reuter SE, Phelps MA, Foster DJR. Optimising time samples for determining area under the curve of pharmacokinetic data using non-compartmental analysis. J Pharm Pharmacol. 2019;71:1635-44.

33. Abernethy DR, Greenblatt DJ, Divoll M, Shader RI. Prolonged accumulation of diazepam in obesity. J Clin Pharmacol. 1983;23:369-76.

34. Smit C, De Hoogd S, Brüggemann RJM, Knibbe CAJ. Obesity and drug pharmacology: a review of the influence of obesity on pharmacokinetic and pharmacodynamic parameters. Expert Opin Drug Metab Toxicol. 2018;14:275-85.

35. Rodieux F, Wilbaux M, van den Anker JN, Pfister M. Effect of kidney function on drug kinetics and dosing in neonates, infants, and children. Clin Pharmacokinet. 2015;54:1183-204.

36. Medicines Development for Global Health. Moxidectin package insert. 2018. https://www.accessdata.fda.gov/drugsatfda_docs/ nda/2018/210867Orig1s000TOC.cfm 